\title{
Use of Surveillance Biopsies in Pediatric Renal Transplant Recipients
}

\author{
Erica D. Winnicki* and Lavjay Butani \\ Section of Pediatric Nephrology, University of California Davis, Sacramento, CA, USA
}

The term 'surveillance biopsy', also often described as 'protocol' biopsy, refers to biopsies that are performed at pre-determined time points after renal transplantation in patients with stable allograft function in order to detect subclinical insults to the allograft, with the intent of early intervention so as to prolong allograft survival. Primarily, surveillance biopsies have been used to detect Sub Clinical Rejection (SCR), defined as histologic evidence of rejection in the absence of any clinical or biochemical signs of graft dysfunction [1]. Other potential benefits include early identification of acute humoral rejection in patients with known high donor specific antibodies, calcineurininhibitor toxicity and other post-transplant complications such as BK virus nephropathy and recurrent disease, all of which may be amenable to intervention. Perhaps due to concerns related to the potential risks associated with the biopsy procedure, especially in the setting of a wellfunctioning allograft, surveillance biopsies have not been universally accepted as a standard of care. Here, we briefly review the literature and attempt to answer the question: should surveillance biopsies be part of routine post-transplant care in pediatric renal transplant recipients?

Initial studies on the use of surveillance biopsies in adult renal transplant recipients reported a high prevalence of SCR. Data from the Winnipeg Transplant Group nearly two decades ago showed that $30 \%$ of biopsies performed within the first 3 months post-transplant in adult recipients on a steroid-based immunosuppressive regimen with cyclosporine and azathioprine satisfied Banff criteria for Type I rejection [2]. Subsequently, the group demonstrated, for the first time, evidence for improved short term outcomes with the use of surveillance biopsies. In their study, patients were randomized to undergo surveillance biopsies at 1,2, 3, 6 and 12 months posttransplant and compared to controls who underwent biopsies at 6 and 12 months or for clinical indication only; patients who were noted to have SCR received corticosteroid treatment. At 24 months after transplantation, compared to the control group, the early surveillance biopsy cohort had a significantly lower serum creatinine concentration [3]. Data from serial surveillance biopsies has also provided evidence that SCR is associated with the development of Interstitial Fibrosis and Tubular Atrophy (IFTA) of the allograft [3,4]. The presence of IFTA, in turn, is associated with decreased long-term graft survival; the presence of both IFTA and SCR, even if inflammation is mild, has been shown to be associated with worse graft survival than in the setting of IFTA alone [5,6]. IFTA can also be seen as a consequence of chronic calcineurin-inhibitor toxicity; several studies have suggested that early identification of IFTA and changing to a non-calcineurin inhibitor based immunosuppressive regimen (such as sirolimus), may slow the progression of graft fibrosis [7].

However, more recent studies in adult transplant recipients receiving contemporary immunosuppressive regimens have not shown a consistent benefit from the use of surveillance biopsies. Rush et al. performed a second randomized trial in adult recipients receiving steroid based immunosuppression with tacrolimus and mycophenolate mofetil. Recipients were randomized to surveillance biopsy at 1, 2, 3 and 6 months versus a 6 month biopsy alone [8]. In this study, the prevalence of SCR was substantially lower than in previous reports $(4.6 \%$ at 3 months). At 6 months post-transplant there was no difference in SCR, IFTA or serum creatinine concentration in those who underwent surveillance biopsies within the first 3 months as compared to controls. The low prevalence of SCR in this cohort presumably explains the lack of benefit of surveillance biopsies. However even in cohorts with a higher prevalence of SCR, the data are conflicting. Scholten et al, in a study that randomized recipients to the use of either cyclosporine or tacrolimus, identified SCR in 31\% of surveillance biopsies at 6 months post-transplant; by design, the SCR was not treated. In this cohort, untreated SCR at 6 months (largely borderline in nature) was not associated with increased IFTA at 12 months or worse renal function at 2 years compared to those without SCR [9]. In contrast, Szederkenyi et al. randomized recipients to surveillance biopsies at either 3 and/or 12 months versus biopsies for clinical indication only. SCR rates were higher in this cohort (seen in nearly half of all surveillance biopsies) with borderline changes accounting for $65 \%$ of those with SCR. Patients with SCR, with the exception of those who had borderline changes, were treated with corticosteroids. Also notable is that calcineurininhibitor toxicity was identified in $21 \%$ of biopsies resulting in dose adjustment or conversion to alternative immunosuppressive therapy. The investigators found improved estimated glomerular filtration rate 3 years post-transplant as well as improved allograft survival 5 years post-transplant in the biopsy arm [10].

Children may be less likely to manifest clinical signs of acute rejection, and as such screening for SCR may be particularly important in this population. It has been shown that children with acute rejection may not have an elevation in their serum creatinine concentration. This has long been postulated to be related to the size mismatch between recipient and donor [11]. Bunchman et al. reported that only $45 \%$ of young children less than 5 years of age had an elevation in their serum creatinine concentration at the time an acute rejection episode was diagnosed [12]. In this study other clinical indicators, such as hypertension and unexplained fever, were used as criteria for biopsy. Similarly, Birk et al. performed serial surveillance biopsies over a 36 month time period post-transplant in a cohort of 21 pediatric renal transplant recipients and found that nearly one third of Banff grade Ia or higher acute rejection episodes diagnosed in this cohort were subclinical [13].

The prevalence of SCR in the first year post-transplant is reported to be high in children. Hymes et al. showed that $29 \%$ of pediatric renal transplant recipients had SCR at 3 months under steroid based maintenance immunosuppression with tacrolimus and mycophenolate mofetil [14]. Similarly, Seikku et al. reported a 39\% prevalence of SCR on 3 month surveillance biopsies in a small group of pediatric patients [15]. At 6 months, the prevalence of SCR was reported to be $25 \%$ [16] and in a different study, 34\% of children had SCR on 12 month surveillance biopsies [17].

*Corresponding author: Erica D. Winnicki, Section of Pediatric Nephrology, University of California Davis, Stockton Blvd, Sacramento, CA95817, USA; Tel: 916-734-8118; Fax: 916-734-0629; E-mail: Erica.winnicki@ucdmc.ucdavis.edu

Received October 14, 2013; Accepted October 15, 2013; Published October 17 2013

Citation: Winnicki ED, Butani L (2013) Use of Surveillance Biopsies in Pediatric Renal Transplant Recipients. J Transplant Technol Res 3: e124. doi:10.4172/2161$0991.1000 \mathrm{e} 124$

Copyright: (c) 2013 Winnicki ED, et al. This is an open-access article distributed under the terms of the Creative Commons Attribution License, which permits unrestricted use, distribution, and reproduction in any medium, provided the original author and source are credited. 
While randomized studies have not been performed to determine whether allograft outcomes are improved as a result of surveillance biopsies in children, there is evidence to suggest that surveillance biopsies and the treatment of SCR in children may be beneficial. In children as in adults, subclinical inflammation has been associated with the development and progression of IFTA [13,18]. Shishido et al. examined the impact of late SCR on the progression of IFTA. Sequential biopsies were performed at 2, 3 and 5 years in living donor pediatric renal transplant recipients who had evidence of IFTA on 1 year surveillance biopsy. Those recipients with IFTA and SCR were more likely to have progression of IFTA on subsequent biopsies; moreover, those with repeated SCR on surveillance biopsy had significantly worse 5 year creatinine clearance and poorer long-term graft survival [17]. Hymes et al. found that SCR, when treated at 3 months post-transplant, did not adversely impact renal function or increase risk of subsequent clinical rejection episodes during a mean follow-up time of 33 months [14].

Some of the hesitation in performing surveillance biopsies in children is related to the perceived risks associated with biopsies of stable allografts. However, adverse outcomes related to the use of surveillance biopsies are rare. Two relatively large single-center retrospective studies of ultrasound guided percutaneous biopsies of renal allografts in children did not report any serious adverse events such as the need for blood transfusion or surgical intervention secondary to the biopsy procedure $[19,20]$. The most common adverse events included macroscopic hematuria and perinephric hematomas; the incidence of both was higher with the use of 16-gauge as compared to 18-gauge biopsy needles. Additionally, the incidence of adverse events was not higher for transperitoneally placed grafts as compared to extraperitoneal kidneys [19].

In conclusion, the use of surveillance biopsies and the treatment of SCR have probable benefit on renal allograft outcomes and the risks are minimal. Children may be less likely to present with clinical signs of rejection relative to adults and thus may have more to gain from the use of surveillance biopsies as part of routine post-transplant monitoring. The optimal timing of surveillance biopsies, as well as the real impact of these biopsies on long-term allograft function, remains to be determined.

\section{References}

1. Rush DN, Nickerson P, Jeffery JR, McKenna RM, Grimm PC, et al. (1998) Protocol biopsies in renal transplantation: research tool or clinically useful? Curr Opin Nephrol Hypertens 7: 691-694.

2. Rush DN, Jeffery JR, Gough J (1994) Protocol biopsies in stable renal transplant patients under triple immunosuppression: results at 6 months. Transplant Proc 26: 2576.

3. Rush D, Nickerson P, Gough J, McKenna R, Grimm P, et al. (1998) Beneficial effects of treatment of early subclinical rejection: a randomized study. J Am Soc Nephrol 9: 2129-2134.

4. Nankivell BJ, Borrows RJ, Fung CL, O'Connell PJ, Allen RD, et al. (2003) The natural history of chronic allograft nephropathy. N Engl J Med 349: 2326-2333.

5. Moreso F, Ibernon M, Gomà M, Carrera M, Fulladosa X, et al. (2006) Subclinical rejection associated with chronic allograft nephropathy in protocol biopsies as a risk factor for late graft loss. Am J Transplant 6: 747-752.
6. Park WD, Griffin MD, Cornell LD, Cosio FG, Stegall MD (2010) Fibrosis with inflammation at one year predicts transplant functional decline. J Am Soc Nephrol 21: 1987-1997.

7. Henderson LK, Nankivell BJ, Chapman JR (2011) Surveillance protocol kidney transplant biopsies: their evolving role in clinical practice. Am J Transplant 11: 1570-1575.

8. Rush D, Arlen D, Boucher A, Busque S, Cockfield SM, et al. (2007) Lack of benefit of early protocol biopsies in renal transplant patients receiving TAC and MMF: a randomized study. Am J Transplant 7: 2538-2545.

9. Scholten EM, Rowshani AT, Cremers S, Bemelman FJ, Eikmans M, et al. (2006) Untreated rejection in 6-month protocol biopsies is not associated with fibrosis in serial biopsies or with loss of graft function. J Am Soc Nephrol 17: 2622-2632.

10. Szederkenyi E, Ivanyi B, Morvay Z, et al. (2011) Treatment of subclinical injuries detected by protocol biopsy improves the long-term kidney allograft function: a single center prospective randomized clinical trial. Transplant Proc 43: 1239-1243.

11. Trompeter RS, Bewick M, Haycock GB, Chantler C (1983) Renal transplantation in very young children. Lancet $1: 373-375$.

12. Bunchman TE, Fryd DS, Sibley RK, Mauer SM (1990) Manifestations of renal allograft rejection in small children receiving adult kidneys. Pediatr Nephrol 4 255-258.

13. Birk PE, Stannard KM, Konrad HB, Blydt-Hansen TD, Ogborn MR, et al. (2004) Surveillance biopsies are superior to functional studies for the diagnosis of acute and chronic renal allograft pathology in children. Pediatr Transplant 8: $29-38$

14. Hymes LC, Warshaw BL, Hennigar RA, Amaral SG, Greenbaum LA. (2009) Prevalence of clinical rejection after surveillance biopsies in pediatric renal transplants: does early subclinical rejection predispose to subsequent rejection episodes? Pediatr Transplant 13: 823-826.

15. Seikku P, Krogerus L, Jalanko $H$, Holmberg C (2005) Better renal function with enhanced immunosuppression and protocol biopsies after kidney transplantation in children. Pediatr Transplant 9: 754-762.

16. Kanzelmeyer NK, Ahlenstiel T, Drube J, Froede K, Kreuzer M, et al. (2010) Protocol biopsy-driven interventions after pediatric renal transplantation. Pediatr Transplant 14: 1012-1018.

17. Shishido S, Asanuma H, Nakai H, Mori Y, Satoh H, et al. (2003) The impact of repeated subclinical acute rejection on the progression of chronic allograft nephropathy. J Am Soc Nephrol 14: 1046-1052.

18. Dart AB, Schall A, Gibson IW, Blydt-Hansen TD, Birk PE (2010) Patterns of chronic injury in pediatric renal allografts. Transplantation 89: 334-340.

19. Vidhun J, Masciandro J, Varich L, Salvatierra O Jr, Sarwal M (2003) Safety and risk stratification of percutaneous biopsies of adult-sized renal allografts in infant and older pediatric recipients. Transplantation 76: 552-557.

20. Birk PE, Blydt-Hansen TD, Dart AB, Kaita LM, Proulx C, et al. (2007) Low incidence of adverse events in outpatient pediatric renal allograft biopsies. Pediatr Transplant 11: 196-200. 